\title{
Absorventes Emergentes para Separação de Gases
}

\author{
Emerging Absorbents for Gas Separation. \\ Gas separation and purification operations \\ are relevant at levels ranging from industrial \\ to societal, since they are necessary to \\ produce everyday items, fuel materials, \\ or for the capture of toxic or greenhouse \\ gases. The importance of these types of \\ operations along with the increasing need \\ to develop sustainable industrial separation \\ technologies has driven research in this \\ field. In this article we will briefly review \\ two absorbents in the field of gas capture \\ and separation: deep eutectic solvents (DES) \\ and porous liquids. We will discuss recent \\ developments, the positive and negative \\ aspects, recommendations, and future \\ perspectives for both types of absorbents.
}

\begin{abstract}
As operações de separação e captura de gases têm relevância a nível industrial e social uma vez que são necessárias para a produção de combustíveis, de materiais usados no dia-a-dia, ou para capturar gases tóxicos, ou gases de efeito estufa. A importância destas operações, a par com a necessidade crescente de desenvolver tecnologias de separação industriais mais sustentáveis, tem impulsionado a pesquisa nesta área. Neste artigo irão ser revistos dois tipos de absorventes com relevância na área da captura e separação de gases: os solventes eutécticos profundos ("Deep Eutectic Solvents" - DES) e os líquidos porosos. Serão discutidos os desenvolvimentos recentes, aspetos positivos e negativos, recomendações e perspetivas futuras para estes dois tipos de absorventes.
\end{abstract}

\begin{abstract}
Introdução
As operações de separação e purificação fazem parte do dia-a-dia da maioria dos químicos e engenheiros químicos, desde a pequena escala a nível dos laboratórios académicos até à escala industrial. Na indústria, estas operações representam entre 40 a $90 \%$ dos custos de capital e custos operacionais [1] e são responsáveis por 10 a $15 \%$ do consumo da energia mundial e emissões equivalentes de gases de efeito estufa [2]. Em geral, os processos de separação baseiam-se nas diferenças nas propriedades das espécies a separar, seja a nível molecular, termodinâmico ou nas propriedades de transporte. A prática de separação mais comum e mais conhecida na indústria é a destilação, que faz uso de diferenças de pressões de vapor dos componentes da mistura para promover a sua separação [1]. No entanto, este é geralmente considerado um processo de baixa eficiência g'lobal, rondando os $11 \%[3,4]$.

A separação de alcanos e alcenos de cadeia curta e a captura de gases de efeito estufa resultantes de emissões são exemplos de processos com grande potencial de otimização $[1,2,4]$. A avaliação da otimização de um
\end{abstract}

processo a nível do seu custo energético e ambiental é complexa e tem de ter em conta a concentração das espécies a separar na mistura inicial e final, taxas de fluxo correspondentes, requisitos de temperatura e pressão, facilidade de aumento da escala, requisitos energéticos, sociais, ambientais e considerações económicas $[3,5]$. Uma análise detalhada do custo e sustentabilidade de qualquer tecnologia de separação tem de ser avaliada no contexto da totalidade do processo. Fatores como o ciclo de vida da tecnología, facilidade e custo de substituição ou manutenção de componentes também têm de ser tomados em consideração [2], fazendo com que a avaliação da otimização de um processo não seja uma tarefa simples.

0 uso de tecnologias baseadas em materiais absorventes ou membranas tem aumentado, e envolve, em muitos casos, agentes de separação específicos, o que permite obter processos suficientemente eficientes e seletivos para superar as entropias de mistura [3]. Estes agentes podem tirar partido das diferenças de tamanho ou forma das moléculas ou ainda das 
interações com os componentes a separar [1]. Algumas tecnologias baseadas nestes princípios alcançaram sucesso a nível industrial, tais como as membranas para a dessalinização da água do mar via osmose inversa [6,7] e líquidos iónicos para remoção de vapores de mercúrio do gás natural, HycaPure Hg'M [8].

As mudanças de paradigma necessárias para combater o aquecimento global, a introdução de nova legislação e o crescimento de preocupações ambientais e sociais têm impulsionado a investigação de novas tecnologias e materiais para operações de separação. Neste artigo serão revistos dois absorventes recentes usados para captura ou separação de gases. Estes são os solventes eutécticos profundos (DES, do inglês Deep Eutectic Solvents) e os líquidos porosos, ambos num nível de maturidade tecnológica abaixo de 4 . Serão incluídos comentários a nível da capacidade destes solventes para a sorção de gás, a sua seletividade para a separação, regeneração, custo e sustentabilidade de produção dos mesmos. 0 objetivo deste artigo não é fornecer uma revisão detalhada e exaustiva das tecnologias disponíveis para estas separações, mas sim de fornecer uma visão geral e didática com exemplos concretos de separações estudadas para cada tipo destes absorventes.

A maioria dos artigos foca-se no estudo da absorção de um gás puro, sendo que o gás mais estudado é o dióxido de carbono $\left(\mathrm{CO}_{2}\right)$. Estudos de absorção com gases puros são cruciais para determinar o potencial industrial de um absorvente, pois permitem compreender o sistema ao nível molecular, determinar a natureza do processo e cinética de sorção, definir a capacidade do solvente para capturar cada gás, analisar efeitos de temperatura e pressão, e aceder a seletividades ideais (a razão das capacidades de sorção para os gáses puros) associadas à separação.

A sorção pode envolver um processo físico ou, adicionalmente, um processo químico, reversível ou irreversível. Materiais comerciais como os peneiros moleculares, DPEG (éter dimetílico de poli(etilenoglicol) ou éter dimetílico de poli(óxido de etileno)) ou PC (carbonato de propileno ou 4-metil-1,3-dioxolan-2-ona) fazem uso da sorção física (Figura 1). 0 uso de soluções aquosas de aminoálcoois, como a mono- ou dietanolamina (MEA, 2-aminoetanol e DEA, 2,2'-iminodietanol) (Figura 1) para a absorção de gases ácidos como o $\mathrm{CO}_{2}$ e o $\mathrm{H}_{2} \mathrm{~S}$ baseia-se numa reação reversível; se o absorvente for uma solução aquosa de hidróxido de sódio, o processo torna-se irreversível. Os processos químicos de sorção oferecem geralmente capacidades e seletividades atraentes, mas envolvem um preço energético mais elevado para a recuperação do gás capturado. Além disso, o DPEG, a MEA e a DEA são compostos voláteis, tóxicos, corrosivos e/ou com baixa estabilidade térmica e/ou oxidativa [9].
Figura 1 - Compostos disponíveis comercialmente usados em processos industriais de absorção de $\mathrm{CO}_{2}$. Em cima, compostos usados para a absorção física, o DPEG (éter dimetílico de poli(etilenoglicol), éter dimetílico de poli(óxido de etileno)) e PC (carbonato de propileno, 4-metil-1,3-dioxolan-2-ona. Em baixo compostos usados para a absorção química reversível, a MEA (monoetanolamina ou 2-aminoetanol) e a DEA (dietanolamina ou 2,2'-iminodietanol).<smiles>COC(C)(C)CCOC(C)(C)C</smiles><smiles>CC1COC(=O)O1</smiles><smiles>NCCO</smiles>

MEA

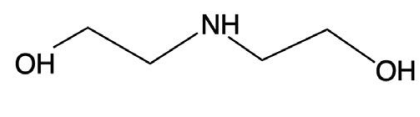

DEA
$\mathrm{O} \mathrm{CO}_{2}$ capturado pode ser utilizado em bebidas gaseificadas ou para promover o crescimento de culturas em estufas. Pode também ser convertido em materiais de valor acrescentado, como químicos, combustíveis ou materiais de construção, como por exemplo, metano, metanol, eteno, carbonatos cíclicos ou policarbonatos [10].

\section{Solventes eutécticos profundos (DES)}

Os solventes eutécticos profundos (DES) são uma classe de compostos que têm recentemente atraído o interesse como solventes na comunidade científica desde que foram popularizados por Abbott em 2003 [11]. 0 número de publicações relacionadas com os DES aumentou de 100 em 2012 para mais de 1350 em 2020. No entanto, as misturas eutécticas tinham sido utilizadas, por exemplo, em produtos farmacêuticos para solubilizar ou liquefazer compostos [12], como materiais de mudança de fase [13] ou em cristais líquidos [14].

As misturas eutécticas são produzidas quando dois ou mais componentes, geralmente constituídos por ácidos e bases de Lewis ou Brønsted, formam uma mistura homogénea para a qual a temperatura de fusão é reduzida quando comparada com a temperatura de fusão dos componentes individuais. Estritamente falando, os DES são misturas eutécticas para as quais a temperatura eutéctica é substancialmente menor que a correspondente para a mistura líquida ideal [17]. Além disso, a composição eutéctica corresponde somente à mistura com a temperatura de fusão mais baixa [18]. A redução do ponto de fusão nos DES tem sido atribuída à formação de interações por pontes de hidrogénio, interações de van der Waals e entropias de mistura $[15,16]$. No entanto, a denominação DES 
é geralmente aceite para uma variedade de líquidos homogéneos fora destas restrições. Dado que a grande maioria dos estudos não apresenta o comportamento ou diagrama de fases do solvente, para o propósito deste artigo não haverá distinção entre os DES e outras misturas com baixo ponto de fusão.

Os DES são geralmente fáceis de preparar e requerem somente a mistura de componentes que são frequentemente comerciais e/ou naturais. Os métodos de preparação mais populares incluem o uso de calor, moagem, adição de solvente ou extrusão [19]. Outras vantagens que os DES podem apresentar são o baixo custo, a volatilidade reduzida, a baixa toxicidade e a facilidade em modular as suas propriedades com diferentes combinações e proporções de uma grande variedade de componentes disponíveis. Esta variedade torna a generalização das propriedades dos DES difícil devido à existência de possíveis efeitos sinergísticos. Será sempre necessária a caracterização individual e exaustiva de cada sistema [20-23].

Os DES são ainda o foco de estudos em áreas como a catálise, o processamento de metais, o tratamento de biomassa, a síntese de polímeros e as operações de extração e separação, incluindo gases e compostos orgânicos voláteis [15,24-27]. Mais recentemente, vários autores dedicaram-se à compreensão destes sistemas a nível da sua estrutura molecular [28,29], robustez, biodegradabilidade [19,30] e volatilidade [21]. Os DES são tradicionalmente classificados em cinco tipos ( I a V): Tipo I - sal quaternário de amónio + cloretos metálicos; Tipo II - sal quaternário de amónio + hidratos de cloretos metálicos; Tipo III - sal quaternário de amónio + doador de pontes de hidrogénio; Tipo IV - hidratos de cloretos metálicos + doador de pontes de hidrogénio; Tipo V (recente) - espécies não iónicas [26,31].

O tipo de DES mais estudado será possivelmente o III, sendo que o arquétipo dos DES é o cloreto de colina (ChCl, (2-hidroxietil)-trimetilamónio), um sal quaternário de amónio combinado com a ureia (U) na proporção de 1:2. Exemplos de componentes estão representados na Figura 2.

Mais recentemente surgiu um novo tipo de DES, com capacidades supramoleculares, constituído pela B-ciclodextrina (Figura 3) e o ácido levulínico (Lev, ácido 4-oxopentanóico) [32,33]. As ciclodextrinas são compostos derivados do amido, usados nas indústrias farmacêutica, cosmética e alimentar pelas suas capacidades em formar complexos de inclusão com moléculas hóspede [34], apesar da sua baixa solubilidade. A incorporação da $\beta$-ciclodextrina como componente do DES evita este problema. O DES resultante retém a capacidade de formar complexos de inclusão, tornando-o um DES com capacidades supramoleculares.

Figura 2 - Exemplos de componentes de DES.

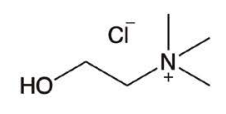

Cloreto de colina, $\mathrm{ChCl}$<smiles>NC(N)=O</smiles>

Ureia, U<smiles>CCC(=O)CCC(=O)O</smiles>

Ácido levulínico<smiles>OCC(O)CO</smiles>

Glicerol
$\mathrm{HO}$<smiles>OCCO</smiles>

Etilenoglicol<smiles>c1c[nH]nn1</smiles>

1,2,3-Triazole<smiles>Oc1cccc(O)c1</smiles>

Resorcinol

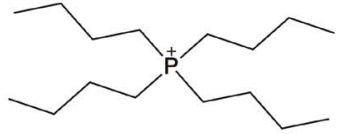

$\mathrm{Br}$

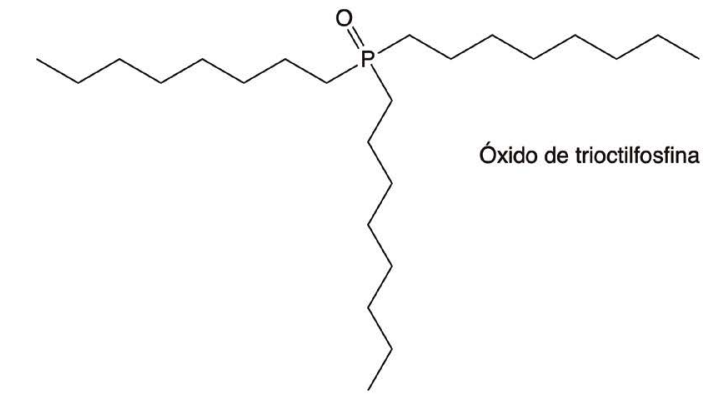

QUímICA | Vol. 45 | N. ${ }^{\circ} 161 \mid 2021$ | 123 

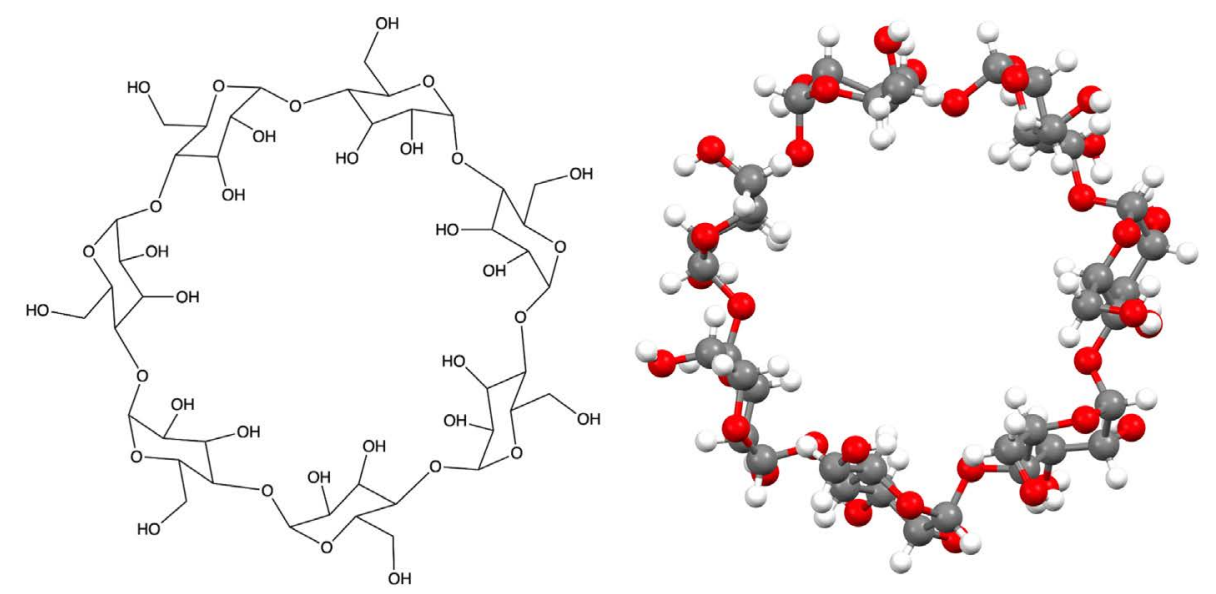

Figura 3 - À esquerda a $\beta$-ciclodextrina e à

direita o seu modelo $3 \mathrm{D}$ em que os átomos a cinzento representam o carbono, os átomos a vermelho o oxigénio e os átomos a branco o hidrogénio.

Relativamente à separação ou captura de gases com DES, o gás mais estudado é o dióxido de carbono $\left(\mathrm{CO}_{2}\right)$, seguido do dióxido de enxofre $\left(\mathrm{SO}_{2}\right)$ e do amoníaco $\left(\mathrm{NH}_{3}\right)$. Outros gases, menos estudados, são o monóxido de carbono (CO), o óxido nítrico (NO), o dióxido de nitrogénio $\left(\mathrm{NO}_{2}\right)$, o hidrogénio $\left(\mathrm{H}_{2}\right)$, o sulfureto de hidrogénio $\left(\mathrm{H}_{2} \mathrm{~S}\right)$, o nitrogénio $\left(\mathrm{N}_{2}\right)$ e o metano $\left(\mathrm{CH}_{4}\right)$ [35].

Um exemplo estudado é o da separação $\mathrm{CO}_{2} /$ $\mathrm{CH}_{4}$, com relevância para a purificação de biogás a biometano, um gás combustível de origem renovável. As condições de purificação do biogás implicam pressões entre 1 e 8 bar, frações molares de $\mathrm{CO}_{2}$ entre 0,15 e 0,5 e temperaturas entre os 283 e os $313 \mathrm{~K}$. 0 biogás contém um número elevado de impurezas, tal como o $\mathrm{H}_{2} \mathrm{~S}$ e $\mathrm{NH}_{3}$. A sua eliminação requer passos de pré-purificação já que muitas das tecnologias atuais não são compatíveis com estas impurezas [36]. De maneira a cumprir a norma de qualidade da União Europeia para o gás natural, o biometano tem de conter mais de $96 \%$ em $\mathrm{CH}_{4}$, entre 2,5 a $4 \%$ de $\mathrm{CO}_{2}$ e outros contaminantes baseados em enxofre ou siloxanos presentes em quantidades abaixo dos 10 $\mathrm{mg} / \mathrm{m}^{3}$ e $0,1 \mathrm{mg} / \mathrm{m}^{3}$, respetivamente [37].

$\mathrm{O}$ DES mais estudado para a separação $\mathrm{CO}_{2} /$ $\mathrm{CH}_{4}$ é o $\mathrm{ChCl:U}(1: 2)$, cujo mecanismo de absorção se baseia num processo físico. Os valores de solubilidade de ambos os gases neste DES são apresentados na Tabela 1, em comparação com os valores para o absorvente físico mais usado na indústria, a água. Há uma grande variação nos valores de solubilidade encontrados na literatura, uma variação maior que as incertezas dos métodos experimentais associados (1-9\%). Isto poderá estar relacionado com a sua pureza ou quantidade de água presente nas amostras, dados só presentes em alguns dos artigos e com uma grande gama de valores, entre 0,01 e 2,4\% em massa. Um outro fator de relevância é o método de produção, já que a maioria das amostras foram preparadas entre os 343 e 353 K, próximo da temperatura de decomposição deste DES, $358 \mathrm{~K}[30,38]$.

Tabela 1 - Valores experimentais de solubilidade de $\mathrm{CO}_{2}$ e $\mathrm{CH}_{4}$ no DES ChCl:U (1:2) [38-44] e em água [45-47] a uma temperatura de $313 \mathrm{~K}$ (ou $308 \mathrm{~K}$ quando assinalado por $\left.{ }^{*}\right)$ e à pressão de 1 bar. Solubilidades expressas em percentagem de massa de gás na mistura $\left(\%\right.$ massa $\left._{\text {gás }}\right)$, em moles de gás capturadas por mole de solvente $\left(n_{\mathrm{gas}}\right)$. A seletividade ideal para a separação $\left(\alpha_{\mathrm{CO}_{2} / \mathrm{CH}_{4}}\right)$ foi calculada como a razão das frações molares de $\mathrm{CO}_{2}$ e CH obtidos nas mesmas condições com gases puros.

\begin{tabular}{|c|c|c|c|c|c|}
\hline Sistema & $\%$ massa & $\mathrm{CO}_{2}$ & $\%$ massa $_{\mathrm{CH}_{4}}$ & $\mathrm{n}_{\mathrm{CO}_{2}} \times 10^{-4}$ & $\mathrm{n}_{\mathrm{CH}_{4}} \times 10^{-4}$ \\
\hline ChCl:U (1:2) & $0,11-0,36$ & $0,0034-0,046^{*}$ & $22-82$ & $2,0-2,5$ & $3,5^{*}-15$ \\
\hline Água & $0,10-0,12^{*}$ & $0,016^{*}-0,018$ & $4,2-4,7^{*}$ & $1,8^{*}-2,1$ & $2,0-2,7^{*}$ \\
\hline
\end{tabular}

Notas: Os DES são misturas não ideais, ou seja, a sua massa molar foi calculada segundo a definição termodinâmica, como a soma das massas molares dos componentes. Para ChCl:U (1:2) o valor é $86,58 \mathrm{~g} \mathrm{~mol}^{-1}$. Solubilidades calculadas a partir das constantes de Henry. No caso da solubilidade dos gases em água foram usadas as equações recomendadas pelos autores para o cálculo das constantes de Henry às temperaturas de 308 e $313 \mathrm{~K}$. 
Apesar das variações, quando se comparam os valores de solubilidade, verifica-se que o DES tem uma afinidade molar entre 5 e 20 vezes mais elevada que a água para o $\mathrm{CO}_{2}$. As afinidades da água e do $\mathrm{DES}$ pelo $\mathrm{CH}_{4}$ são semelhantes. Como resultado, as seletividades de separação são até seis vezes mais elevadas para o DES.

Os grupos de Ji e Liu $[38,48-50]$ e Haghbakhsh e Raeissi [51] analisaram o potencial deste DES e das suas soluções aquosas quando comparado com processos físicos usados na indústria, a água, o DPEG e o PC. Os autores utilizaram uma variedade de parâmetros, tal como a capacidade de absorção, seletividade, pureza da mistura de gás final, efeito do fluxo e composição do gás, tamanho do equipamento de separação, reciclabilidade e perda de solvente, coeficiente de transferência de massa, capacidade de produção de biogás, impacto ambiental e o custo anual total incluindo o custo da energia e materiais. Foram também incluídos dados experimentais da solubilidade dos gases, modelização dos sistemas, simulações e análises termodinâmicas dos processos com o software Aspen Plus ${ }^{\circledR}$. Os autores concluíram que as soluções aquosas de DES têm performances superiores às da água e do DPEG em termos de capacidade de absorção de $\mathrm{CO}_{2}$, seletividade para a separação $\mathrm{CO}_{2} / \mathrm{CH}_{4}$, capacidade de produção de biogás, quantidade de solvente necessário, utilização de energia (diminuição entre 11-16\%), tamanho do equipamento de separação, custo de materiais e custo anual total. Os valores encontrados para o DES são comparáveis ao processo usando PC, com a vantagem do primeiro ser mais benigno. 0 uso de absorventes físicos baseados em DES com uma maior capacidade de absorção de $\mathrm{CO}_{2}$ e menor viscosidade tornará a sua utilização ainda mais vantajosa em relação ao PC [38,48-50].

De maneira a explicar as diferenças de solubilidade do $\mathrm{CO}_{2}$ e do $\mathrm{CH}_{4}$ no DES ChCl:U (1:2), Huang et al. [39] usaram simulações Monte Carlo. Os resultados apontam para a formação de interações por pontes de hidrogénio fracas entre $\mathrm{ChCl}$ e o $\mathrm{CO}_{2}$ e indicam interações fracas do tipo van der Waals com o gás mais inerte, $\mathrm{CH}_{4}$. No entanto, os autores consideram que o fator dominante seja o volume livre do solvente. As diferenças de entalpias de solvatação dos dois gases combinam com as interações mais fortes observadas com o $\mathrm{CO}_{2}$.

Seria de grande interesse ter acesso às contribuições entálpicas e entrópicas da solvatação de ambos os gases numa seleção de DES, simulações de dinâmica molecular [52] e medidas experimentais de dispersão de neutrões [53], além de medidas de solubilidades com gases mistos. Estes resultados ajudariam a compreender melhor as interações com cada gás, os mecanismos de absorção e a influência da presença de contaminantes.

A adição de grupos amina é uma estratégia utilizada frequentemente para aumentar a capacidade de captura de $\mathrm{CO}_{2}$ por meio de uma reação reversível. As aminas reagem com o CO 2 através da formação do (ou derivado do) ácido carbâmico ou do sal de carbamato -RR'NCOO- e/ou carbonatos na presença de água [54]. Esta abordagem foi aplicada nos líquidos iónicos [55], hidrogéis [56], polímeros porosos [57-59], materiais inorgânicos como a sílica (dióxido de silício) [60] ou óxidos metálicos [61], estruturas metal-orgânicas (MOFs, Metal Organic Frameworks) [62], estruturas orgânicas covalentes (COFs, Covalent Organic Frameworks) [63] e também em DES [64-68]. Por exemplo, Trivedi et al. [64] produziram um DES, [MEA.CI][EDA] (1:3) (Figura 4) que proporcionou uma grande capacidade de captura de $\mathrm{CO}_{2}$ em massa ( $31,5 \%$ ou 0,54 moles de $\mathrm{CO}_{2}$ por mol de solvente a 1 bar e $303 \mathrm{~K}$ ). Este DES apresenta capacidades de absorção competitivas quando comparadas com o absorvente químico convencional, uma solução aquosa de MEA a 30\% em massa, que apresenta uma absorção em massa entre os 12 e 13\%, ou aproximadamente 0,68 moles por mole de amina [69]. A cinética de absorção inicial do DES é também promissora (25,2\% em massa após 3 h a 303 K), com boa reciclabilidade, tolerância à presença de água e ao aumento de temperatura. Este DES permite evitar algumas das desvantagens relacionadas com o uso de aminas convencionais, tais como ental pias de absorção 28\% mais favoráveis (facilitando a regeneração), e maior resistência à corrosão devido à rede de pontes de hidrogénio presentes no DES. O novo solvente apresenta também algumas desvantagens, tal como um aumento da viscosidade da mistura de quase 185 vezes após absorção do $\mathrm{CO}_{2}$ [64].

Estes solventes demonstram trazer certas vantagens como a facilidade de modular as suas estruturas para aplicações de absorção e separação de gases (física ou também química), custos comparáveis às tecnologias

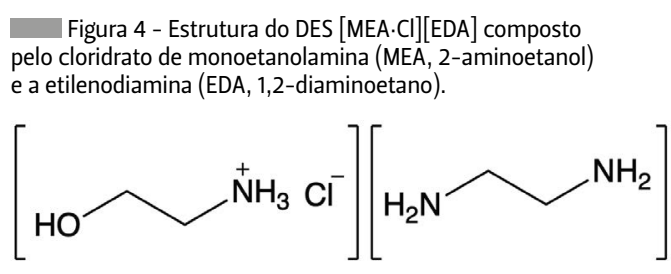

$[\mathrm{MEA} \cdot \mathrm{Cl}][\mathrm{EDA}]$

equivalentes existentes, facilidade de produção e baixa volatilidade. Podem ainda capturar várias impurezas num só passo (ex. $\mathrm{CO}_{2}$ e $\mathrm{H}_{2} \mathrm{~S}$ ). Os DES têm também desvantagens associadas, sendo que frequentemente as viscosidades são relativamente elevadas o que leva a tempos de absorção também elevados. Outra desvantagem são as possíveis baixas temperaturas de degradação.

Possíveis direções na investigaç̧ão poderão ajudar 
a ultrapassar algumas das desvantagens, por exemplo o uso de membranas no caso dos DES [70], suportes sólidos como no caso da HycaPure Hg ${ }^{\mathrm{TM}}$ [8], uso de soluções aquosas de DES, como discutido acima, ou outros co-solventes, além de modificações estruturais dos DES que tragam um aumento da sua capacidade de absorção e diminuição de viscosidade.

\section{Líquidos porosos}

Uma maneira engenhosa de aumentar as capacidades de absorção de gases com materiais líquidos é a introdução de "poros". O conceito de líquidos porosos (porous liquids) foi proposto pela primeira vez em 2007 por James [71] e são definidos como líquidos com "porosidade" permanente, com poros vazios (não ocupados por moléculas de solvente). Um líquido convencional pode apresentar porosidade transiente, associada ao espaço entre as moléculas. Nos líquidos porosos a porosidade está associada a cavidades dentro das moléculas. Os líquidos porosos podem ser classificados de acordo com as propriedades dos seus componentes, como tipos I a III (Figura 5).

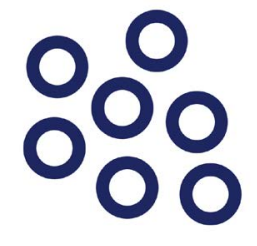

Tipo I

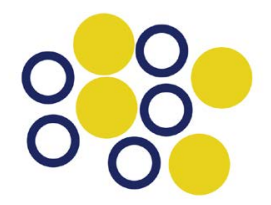

Tipo II

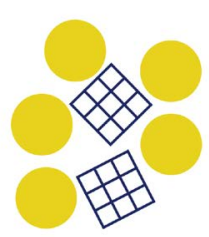

Tipo III
Figura 5 - llustração dos diferentes tipos de líquidos porosos. Tipo I, composto por moléculas com uma cavidade interna vazia; Tipo II, composto por moléculas hospedeiras discretas (a azul, esferas vazias) dispersas num solvente (a amarelo, esferas preenchidas); Tipo
III, composto por partículas de materiais sólidos porosos (a azul matriz) dispersos num solvente (a amarelo, esferas preenchidas). As moléculas de solvente estão estericamente impedidas de preencher as cavidades vazias dos líquidos porosos de Tipos II e III.
Os líquidos porosos de tipo I são compostos por moléculas com uma cavidade interna, rígida e com formas que não permitem autopreenchimento da mesma. Estes líquidos podem ser formados a partir da adição de cadeias alquílicas longas em moléculas porosas como gaiolas orgânicas [72] e esferas vazias de sílica [73]. A adição de cadeias mais longas (> 12 átomos) leva à diminuição do ponto de fusão do material, mas estas cadeias poderão entrar nas cavidades das gaiolas, diminuindo o volume útil para a captura de gases [74,75]. Os líquidos de tipos II e III são geralmente constituídos por uma mistura de material poroso com um solvente com tamanho molecular de modo a que impeça a sua entrada nos poros [76]. No caso dos líquidos de tipo II, a porosidade é obtida a partir de moléculas discretas de hospedeiros porosos, tais como éteres coroa $[77,78]$ ou gaiolas metal-orgânicas (gaiolas de coordenação) [79]. No caso dos de tipo III, a porosidade é gerada por partículas de materiais sólidos porosos, como os zeólitos [80], MOFs [81-83], COFs [84,85] ou silicalite [83]. Entre os solventes escolhidos estão os solventes orgânicos [73,77,81], óleos [85], líquidos iónicos [82-84] e éteres coroa [77-79]. Os líquidos iónicos são frequentemente escolhidos devido às suas baixa volatilidade, estabilidade e variedade estrutural.

Os líquidos porosos atraíram atenção como materiais com potencial para a captura e separação de gases pois permitem tirar partido dos benefícios associados aos sólidos porosos (poros permanentes, rígidos e bem definidos) e aos líquidos (fluidez e melhor transferência de massa e de calor). Testes de absorção de $\mathrm{CH}_{4}$ em líquidos do tipo II, resultados espectroscópicos e simulações moleculares efetuadas em 2015 provaram finalmente a existência e o potencial destes materiais [77,78] e geraram a formação da spin-off "Porous Liquid Technologies Ltd" em 2018. Desde então, os líquidos porosos têm sido testados para a absorção de gases como o CO 2 [80,82,83,87,88], CH $[78,81,82,84,85,87]$, etano e eteno [85,89], propano e propeno [88], $\mathrm{N}_{2}$ $[79,87], \mathrm{H}_{2}$ [81] e Xe [87], sendo que o $\mathrm{CO}_{2}$ é o gás mais estudado. Os autores reportaram valores de absorção de $\mathrm{CO}_{2}$ entre 2 a 20 vezes superiores aos verificados para solventes não porosos, o que corresponde a comportamentos de absorção próximos dos de um material poroso $[84,85]$. Os gases contidos nos poros podem ser recuperados facilmente pela adição de um solvente com moléculas que possam penetrar nos poros e expulsar os gases $[75,77]$ ou, no caso de solventes não-voláteis, pela diminuição da pressão [81].

$\mathrm{Na}$ Tabela 2 são apresentados dados de solubilidade de $\mathrm{CO}_{2}$ e $\mathrm{CH}_{4}$ em alguns dos materiais testados, além da seletividade para a sua separação. Pode ver-se uma gama larga de valores de absorção de $\mathrm{CO}_{2}$, entre 0,085 mmol e 1,14 mmol por grama de líquido poroso, valores várias vezes superiores aos dos solventes não-porosos correspondentes e que se tornam muito mais significativos com o aumento da pressão de gás. De notar o valor mais elevado de seletividade para esta separação em líquidos porosos (144) reportado por Liu et al. [81]. 0 líquido poroso de tipo III usado pelos autores foi obtido misturando três compostos comerciais, ZIF-8 (Figura 6), 2-metilimidazole e glicol [81]. Recentemente, Costa Gomes et al. reportaram os líquidos porosos com melhor performance de captura de $\mathrm{CO}_{2}$ a baixas pressões [86]. Estes líquidos foram formados com misturas de ZIF-8 e o líquido iónico [ $\left.\mathrm{P}_{4444}\right][$ Lev] (levulinato de tetrabutilfosfónio) e são capazes de absorver $\mathrm{CO}_{2}$ através de uma reação química reversível entre o gás e o anião do líquido iónico. 0 resultado é a absorção de 1,14 mmol de $\mathrm{CO}_{2} / g$ de líquido poroso a $303 \mathrm{~K}$ e a 1 bar de pressão ou $1,5 \mathrm{mmol}^{-1}$ a 2 bar, valores na mesma gama de absorções que as etanolaminas comerciais [86]. 
Tabela 2 - Valores experimentais de solubilidade de $\mathrm{CO}_{2}$ e $\mathrm{CH}_{4}$ em líquidos porosos a uma temperatura de $303 \mathrm{~K}$ e a 1 bar. Solubilidades expressas em moles de gás capturadas por grama de líquido poroso $\left(\mathrm{n}_{\text {gás } /} \mathrm{m}_{\mathrm{PL}}\right)$. A seletividade para a separação $\left(\alpha_{\mathrm{CO}_{2} / \mathrm{CH}_{4}}\right)$ foi calculada como a razão das solubilidades de $\mathrm{CO}_{2}$ e $\mathrm{CH}_{4}$ obtidos nas mesmas condições com gases puros.

\begin{tabular}{|c|c|c|c|c|}
\hline Material & $\begin{array}{c}\mathrm{n}_{\mathrm{CO}_{2}} / \mathrm{m}_{\mathrm{PL}} \\
\left.(\mathrm{mmol} \mathrm{g})^{-1}\right)\end{array}$ & $\begin{array}{c}\mathrm{n}_{\mathrm{CH}_{4}} / \mathrm{m}_{\mathrm{PL}} \\
\left(\mathrm{mmol} \mathrm{g}^{-1}\right)\end{array}$ & $\alpha_{\mathrm{CO}_{2} / \mathrm{CH}_{4}}$ & Ref. \\
\hline $\mathrm{ZIF}-8 /\left[\mathrm{P}_{66614}\right]\left[\mathrm{NTf}_{2}\right]$ & 0,085 & 0,034 & 2,5 & [90] \\
\hline PAF1/Genosorb ® & 0,72 & 0,20 & 3,6 & [88] \\
\hline ZIF-8/mimgli & $1,25^{*}$ & - & 144 & {$[81]$} \\
\hline 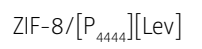 & 1,14 & - & - & \\
\hline
\end{tabular}

Notas: Estrutura do MOF ZIF-8 na Figura 6, $\left[\mathrm{P}_{66614}\right]\left[\mathrm{NTf}_{2}\right]$ é o líquido iónico bis(trifluorometanossulfonil)imida de tri-hexil(tetradecil)fosfónio, $\left[\mathrm{P}_{4444}\right][$ Lev $]$ é o líquido iónico levulinato de tetrabutilfosfónio, mimgli é uma mistura de 2-metilimidazole com glicol, PAF1 é um COF, um polímero orgânico poroso, Genosorb® é uma mistura de DPEGs. *em $\mathrm{mmol} \mathrm{mL}^{-1}$

Para compreender melhor os mecanismos envolvidos na absorção de gases por líquidos porosos, Zhang et al. estudaram a termodinâmica e cinética da sorção de $\mathrm{CH}_{4}, \mathrm{CO}_{2}$ e $\mathrm{N}_{2}$ em líquidos porosos de tipo II, formados por moléculas-gaiola orgânicas (como na Figura 6) funcionalizadas com éteres coroa, dispersas no solvente 15-coroa-5 (1,4,7,10,13-pentaoxaciclopentadecano) [91]. Os autores concluíram que o armazenamento dos gases é governado por interações intermoleculares não eletrostáticas dispersivas com as moléculas gaiola e pela forma/tamanho dos gases e gaiolas. 0 primeiro controla a afinidade dos gases pela gaiola enquanto o segundo determina se o espaço da cavidade é usado eficientemente [91].

Os líquidos porosos apresentam também desafios, com melhores performances geralmente a pressões superiores à atmosférica e para pressões parciais elevadas de gás a capturar [92]. Os de tipo I podem ser difíceis de sintetizar, com viscosidades e pontos de fusão elevados [75]. Os de tipos II e III podem ser obtidos mais facilmente pela simples mistura de produtos comerciais, mas em muitos casos apresentam preços elevados e/ou sínteses complexas ou com muitos desperdícios [83]. Pode também ser difícil de obter líquidos estáveis com altas concentrações de material poroso, requerendo modificações dos componentes [78]. Algumas das desvantagens poderão ser contornadas pelo uso de líquidos porosos na forma de membranas $[81,85,88]$. A funcionalização da superfície dos MOF, para obter maior controlo no tamanho dos poros e na afinidade para o solvente e o gás, poderá constituir uma linha de investig’ação importante pois poderá levar a uma redução do custo dos materiais e o melhoramento da sustentabilidade destes líquidos usando materiais de origem natural ou obtidos a partir de desperdícios.

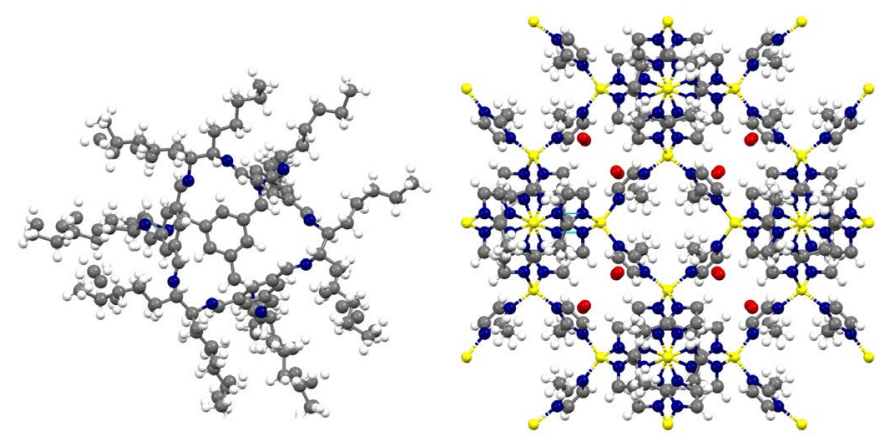

Gaiola orgânica ZIF-8

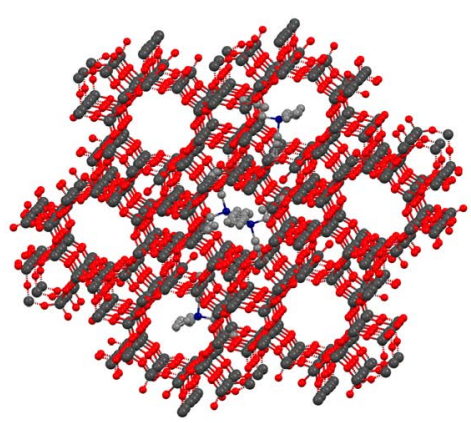

ZSM-5

Figura 6 - Estruturas 3D de materiais porosos usados em líquidos porosos. No topo à esquerda uma gaiola orgânica usada num líquido poroso de tipo I [72]; à direita um MOF usado num líquido poroso de tipo III, ZIF-8 [81,83,86]; em baixo um zeólito usado num líquido poroso de

tipo III, o ZSM-5 [80]. Átomos a cinzento representam carbono, a cinzento-escuro o silício, a vermelho o oxigénio, a branco o hidrogénio, a azul o nitrogénio e a amarelo o zinco. Estruturas obtidas a partir dos ficheiros CIF através da base de dados de cristalografia de Cambridge.

\section{Conclusões}

Mais estudos são necessários para desenvolver uma compreensão mais detalhada dos DES e dos líquidos porosos como sistemas para absorção e separação de gases. No caso dos DES seria benéfico analisar o seu comportamento de fase enquanto solventes puros e em misturas, determinar as estruturas das soluções e das misturas com gases e água, temperaturas de decomposição, volatilidade, ecotoxicidade e biodegradabilidade, estudos experimentais em condições industriais ou 
avaliação dos ciclos de vida dos processos. No caso dos líquidos porosos, e dado que estes são menos estudados que os DES, seria interessante obter mais informação sobre a relação entre as estruturas dos componentes e o comportamento do líquido e obter mais informação relacionada com a termodinâmica, cinética e mecanismos envolvidos na seletividade de absorção de uma variedade de gases.

Os dois absorventes discutidos são recentes e enfrentam vários desafios. Embora estes não apresentem ainda todas as propriedades ideais para a sua aplicação, os estudos descritos demonstram que estas classes de materiais têm um grande potencial para solucionar problemas críticos das tecnologias usadas industrialmente.

\section{Agradecimentos}

A autora agradece o apoio da Royal Academy of Engineering pela sua Research Fellowship de cinco anos. Agradece também o apoio do QUILL (Queen's University lonic Liquid Laboratories) e da Queen's University Belfast no desenvolvimento do seu grupo de investigação. A autora reconhece também a discussão e apoio dos seus colaboradores, Prof. John Holbrey, Prof. Margarida Costa Gomes, Prof. Sophie Fourmentin, Dr. Gosia Swadzba-Kwasny e Dr. Panagiotis Manesiotis, e dos seus estudantes de doutoramento Sam McCalmont e Mark Young.

\section{Referências}

[1] A. B. de Haan, H. Bosch, "Industrial Separation Processes", Berlin, Boston, De Gruyter, 2013.

[2] D. S. Sholl, R. P. Lively, Nature 2016, 532, 435-437. DOI: 10.1038/532435a.

[3] E. L. Cussler, B. K. Dutta, AlChE J. 2012, 58, 3825-3831. DOI: 10.1002/aic.13779.

[4] C. Dollinger, J. Cresko, S. Brueske, A. Carpenter, "Bandwidth study on energy use and potential energy saving opportunities in manufacturing food and beverages," United States, 2016. [Online]: osti.gov/biblio/1513859.

[5] E. J. Henley, J. D. Seader, D. K. Roper, "Separation Process Principles", Wiley, 2011.

[6] N. M. Wade, Desalination 2001, 136, 3-12. DOI: 10.1016/S00119164(01)00159-X.

[7] S. A. Avlonitis, K. Kouroumbas, N. Vlachakis, Desalination 2003, 157, 151158. DOI: 10.1016/S0011-9164(03)00395-3.

[8] M. Abai, M. P. Atkins, A. Hassan, J. D. Holbrey, Y. Kuah, P. Nockemann, A. A. Oliferenko, N. V. Plechkova, S. Rafeen, A. A. Rahman, R. Ramli, S. M. Shariff, K. R. Seddon, G. Srinivasan, Y. Zoub, Dalton Trans. 2015, 44, 8617-8624. DOI: 10.1039/C4DT03273」.

[9] M. Wei, A. C. Huang, C. M. Shu, L. Zhang, Sci. Rep. 2019, 9, 1-9. D0l: 10.1038/ s41598-018-38434-1.

[10] O. S. Bushuyev, P. De Luna, C. T. Dinh, L. Tao, G. Saur, J. van de Lagemaat, S. O. Kelley, E. H. Sargent, Joule 2018, 2, 825-832. DOI: 10.1016/j.joule.2017.09.003.

[11] A. P. Abbott, G. Capper, D. L. Davies, R. K. Rasheed, V. Tambyrajah, Chem. Commun. 2003, 1, 70-71. DOI: 10.1039/b210714g.

[12] A. Pelczarska, D. Ramjugernath, J. Rarey, U. Domańska, J. Chem. Thermodyn 2013, 62, 118-129. DOI: 10.1016/j.jct.2013.02.007.

[13] D. Wei, S. Han, B. Wang, Fluid Phase Equilib. 2014, 373, 84-88. DOI: 10.1016/j.fluid.2014.04.020.

[14] A. E. Hoyt, S. J. Huang, J. Macromol. Sci. Part A 1995, 32, 1931-1945, DOI: 10.1080/10601329508009371.

[15] E. L. Smith, A. P. Abbott, K. S. Ryder, Chem. Rev. 2014, 114, 11060-11082. DOI: $10.1021 / \mathrm{cr} 300162 p$

[16] M. Francisco, A. van den Bruinhorst, M. C. Kroon, Angew. Chem. Int. Ed. 2013, 52, 3074-3085. DOI: 10.1002/anie.201207548.

[17] M. A. R. Martins, S. P. Pinho, J. A. P. Coutinho, J. Solution Chem. 2019, 48, 962-982. DOI: 10.1007/s10953-018-0793-1.

[18] P. Atkins, J. De Paula, "Atkins' Physical Chemistry" $9^{\text {th }}$ ed., Oxford University Press, New York, 2006.

[19] N. R. Rodriguez, A. van den Bruinhorst, L. J. B. M. Kollau, M. C. Kroon, K. Binnemans, ACS Sustain. Chem. Eng. 2019, 7, 11521-11528. DOI: 10.1021/ acssuschemeng.9b01378.

[20] M. Hayyan, M. A. Hashim, A. Hayyan, M. A. Al-Saadi, I. M. AlNashef, M. E. S. Mirghani, O. K. Saheed, Chemosphere 2013, 90, 2193-2195. DOI 10.1016/j.chemosphere.2012.11.004.

[21] C. H. J. T. Dietz, J. T. Creemers, M. A. Meuleman, C. Held, G. Sadowski, M. van S. Annaland, F. Gallucci, M. C. Kroon, ACS Sustain. Chem. Eng. 2019, 7, 40474057. DOI: 10.1021/acssuschemeng.8b05449.

[22] Q. Wen, J. X. Chen, Y. L. Tang, J. Wang, Z. Yang, Chemosphere 2015, 132, 6369. DOI: $10.1016 /$ j.chemosphere.2015.02.061.

[23] T. El Achkar, H. G.-Gerges, S. Fourmentin, "Understanding the Basics and Properties of Deep Eutectic Solvents BT - Deep Eutectic Solvents for Medicine, Gas Solubilization and Extraction of Natural Substances", S. Fourmentin, M. Costa Gomes, E. Lichtfouse (Eds.), Cham: Springer International Publishing, 2021

[24] Q. Zhang, K. de O. Vigier, S. Royer, F. Jérôme, Chem. Soc. Rev. 2012, 41, 7108-7146. DOI: 10.1039/c2cs35178a.

[25] E. L. Byrne, R. O'Donnell, M. Gilmore, N. Artioli, J. D. Holbrey, M. S.-Kwaśny, Phys. Chem. Chem. Phys. 2020, 22, 24744-24763. DOl: 10.1039/d0cp02605k.

[26] M. Gilmore, É. N. McCourt, F. Connolly, P. Nockemann, M. S.-Kwaśny, J. D. Holbrey, ACS Sustain. Chem. Eng. 2018, 6, 17323-17332. DOI: 10.1021/ acssuschemeng.8b04843.

[27] S. Fourmentin, M. Costa Gomes, E. Lichtfouse, "Deep Eutectic Solvents for Medicine, Gas Solubilization and Extraction of Natural Substances", $1^{\text {st }}$ ed. Springer International Publishing AG, 2020.

[28] O. S. Hammond, D. T. Bowron, K. J. Edler, Green Chem. 2016, 18, 2736-2744. DOl: 10.1039/c5gc02914g.

[29] M. Gilmore, L. M. Moura, A. H. Turner, M. S.-Kwaśny, S. K. Callear, J. A. McCune, O. A. Scherman, J. D. Holbrey, J. Chem. Phys. 2018, 148, 193823. DOI: $10.1063 / 1.5010246$

[30] M. Gilmore, M. Swadzba-Kwasny, J. D. Holbrey, J. Chem. Eng. Data 2019, 64, 5248-5255. DOI: 10.1021/acs.jced.9b00474.

[31] D. O. Abranches, M. A. R. Martins, L. P. Silva, N. Schaeffer, S. P. Pinho, J. A. P. Coutinho, Chem. Commun. 2019, 55, 10253-10256. D0I: 10.1039/ C9CC04846D

[32] T. El Achkar, L. Moura, T. Moufawad, S. Ruellan, S. Panda, S. Longuemart, F.X. Legrand, M. C. Gomes, D. Landy, H. G.-Gerges, S. Fourmentin, Int. J. Pharm. 2020, 584, 119443. DOI: 10.1016/j.jppharm.2020.119443.

[33] T. El Achkar, T. Moufawad, S. Ruellan, D. Landy, H. G.-Gerges, S. Fourmentin, Chem. Commun. 2020, 56, 3385-3388. DOI: 10.1039/DOCC00460J.

[34] J. Wankar, N. G. Kotla, S. Gera, S. Rasala, A. Pandit, Y. A. Rochev, Adv. Funct. Mater. 2020, 30, 1909049. DOI: 10.1002/adfm.201909049.

[35] L. Moura, L. Kollau, M. C. Gomes, "Solubility of Gases in Deep Eutectic Solvents BT - Deep Eutectic Solvents for Medicine, Gas Solubilization and Extraction of Natural Substances," S. Fourmentin, M. Costa Gomes, E. Lichtfouse (Eds.), Cham: Springer International Publishing, 2021.

[36] F. Ardolino, G. F. Cardamone, F. Parrillo, U. Arena, Renew. Sustain. Energy Rev. 2021, 139, 110588. DOI: 10.1016/j.rser.2020.110588.

[37] E.-E. B. European Bigas Association, "EBA's biomethane fact sheet" 2013

[38] Y. Xie, H. Dong, S. Zhang, X. Lu, X. Ji, Green Energy Environ. 2016, 1, 195-200. DOI: 10.1016/j.gee.2016.09.001.

[39] F. Liu, W. Chen, J. Mi, J.-Yin Zhang, X. Kan, F.-Y. Zhong, K. Huang, A.-M. Zheng, L. Jiang, AIChE J. 2019, 65, e16574. DOI: 10.1002/aic.16574.

[40] R. B. Leron, A. Caparanga, M.-H. Li, J. Taiwan Inst. Chem. Eng. 2013, 44, 879885. DOl: 10.1016/j.jtice.2013.02.005.

[41] X. Li, M. Hou, B. Han, X. Wang, L. Zou, J. Chem. Eng. Data 2008, 53, 548-550. DOI: $10.1021 /$ je700638u. 
[42] N. R. Mirza, N. J. Nicholas, Y. Wu, K. A. Mumford, S. E. Kentish, G. W. Stevens, J. Chem. Eng. Data 2015, 60, 3246-3252. DOI: 10.1021/acs.jced.5b00492.

[43] W. C. Su, D. S. H. Wong, M. H. Li, J. Chem. Eng. Data 2009, 54, 1951-1955. DOI: 10.1021/je900078k

[44] Y. Xie, H. Dong, S. Zhang, X. Lu, X. Ji, J. Chem. Eng. Data 2014, 59, 33443352. DOl: 10.1021/je500320c.

[45] R. Crovetto, J. Phys. Chem. Ref. Data 1991, 20, 575-589. DOl: $10.1063 / 1.555905$

[46] R. Sander, Atmos. Chem. Phys. 2015, 15, 4399-4981. DOI: 10.5194/acp-154399-2015.

[47] R. Crovetto, R. F.-Prini, M. L. Japas, J. Chem. Phys. 1982, 76, 1077-1086. DOl: $10.1063 / 1.443074$

[48] C. Ma, C. Liu, X. Lu, X. Ji, Appl. Energy 2018, 225, 437-447. DOI: 10.1016/j. apenergy.2018.04.112.

[49] C. Ma, C. Liu, X. Lu, X. Ji, Energy Procedia 2017, 142, 3631-3636. DOI 10.1016/j.egypro.2017.12.255.

[50] C. Ma, Y. Xie, X. Ji, C. Liu, X. Lu, Appl. Energy 2018, 229, 1269-1283. DOI: 10.1016/j.apenergy.2017.03.059.

[51] R. Haghbakhsh, S. Raeissi, J. Environ. Chem. Eng. 2019, 7, 103411. DOI: 10.1016/j.jece.2019.103411.

[52] L. Moura, M. Mishra, V. Bernales, P. Fuentealba, A. A. H. Padua, C. C. Santini, M. F. C. Gomes, J. Phys. Chem. B 2013, 117, 7416-7425. DOI: 10.1021/jp403074z.

[53] L. Moura, M. Gilmore, S. K. Callear, T. G. A. Young's, J. D. Holbrey, Mol. Phys. 2019, 117, 3364-3375. DOI: 10.1080/00268976.2019.1649495.

[54] R. B. Said, J. M. Kolle, K. Essalah, B. Tangour, A. Sayari, ACS Omega 2020, 5 , 26125-26133. DOI: 10.1021/acsomega.0c03727.

[55] S. Saravanamurugan, A. J. K.-Kruse, R. Fehrmann, A. Riisager, ChemSusChem 2014, 7, 897-902. DOI: 10.1002/cssc.201300691.

[56] X. Xu, C. Heath, B. Pejcic, C. D. Wood, J. Mater. Chem. A 2018, 6, 4829-4838. DOI: 10.1039/c8ta00602d

[57] P. Jorayev, I. Tashov, V. Rozyyev, T. S. Nguyen, N. A. Dogan, C. T. Yavuz, ChemSusChem 2020, 13, 6433-6441. DOI: 10.1002/CSSC.202002190.

[58] M. M. Abdelnaby, A. M. Alloush, N. A. A. Qasem, B. A. Al-Maythalony, R. B. Mansour, K. E. Cordova, O. C. S. Al Hamouz, J. Mater. Chem. A 2018, 6, 64556462. DOI: 10.1039/c8ta00012c

[59] A. M. Varghese, G. N. Karanikolos, Int. J. Greenh. Gas Control 2020, 96, 103005. DOl: 10.1016/j.jjggc.2020.103005.

[60] S. A. Didas, S. Choi, W. Chaikittisilp, C. W. Jones, Acc. Chem. Res. 2015, 48, 2680-2687. DOI: 10.1021/acs.accounts.5b00284.

[61] X.Zhu, T. Ge, F. Yang, M. Lyu, C. Chen, D. O'Hare, R. Wang, J. Mater. Chem. A 2020, 8, 16421-16428. DOI: 10.1039/d0ta05079b.

[62] R. W. Flaig, T. M. O. Popp, A. M. Fracaroli, E. A. Kapustin, M. J. Kalmutzki, R. M. Altamimi, F. Fathieh, J. A. Reimer, O. M. Yaghi, J. Am. Chem. Soc. 2017, 139, 12125-12128. DOI: 10.1021/jacs.7b06382.

[63] K. Gottschling, L. Stegbauer, G. Savasci, N. A. Prisco, Z. J. Berkson, C. Ochsenfeld, B. F. Chmelka, B. V. Lotsch, Chem. Mater. 2019, 31, 1946-1955. DOI: 10.1021/acs.chemmater.8b04643.

[64] T. J. Trivedi, J. H. Lee, H. J. Lee, Y. K. Jeong, J. W. Choi, Green Chem. 2016, 18, 2834-2842. DOI: 10.1039/c5gc02319j.

[65] H. Ren, S. Lian, X. Wang, Y. Zhang, E. Duan, J. Clean. Prod. 2018, 193, 802 810. DOI: 10.1016/j.jclepro.2018.05.051

[66] K. Zhang, Y. Hou, Y. Wang, K. Wang, S. Ren, W. Wu, Energy and Fuels 2018 , 32, 7727-7733. DOI: 10.1021/acs.energyfuels.8b01129.

[67] I. Adeyemi, M. R. M. Abu-Zahra, I. Alnashef, Energy Procedia 2017, 105, 1394 1400. DOI: 10.1016/j.egypro.2017.03.519.

[68] Z. Li, L. Wang, C. Li, Y. Cui, S. Li, G. Yang, Y. Shen, ACS Sustain. Chem. Eng. 2019, 7, 10403-10414. DOI: 10.1021/acssuschemeng.9b00555.

[69] F.-Y Jou, A. E. Mather, F. D. Otto, Can. J. Chem. Eng. 1995, 73, 140-147. DOI: $10.1002 / c j c e .5450730116$.

[70] R. Craveiro, L. A. Neves, A. R. C. Duarte, A. Paiva, Sep. Purif. Technol. 2021, 254, 117593. DOI: 10.1016/j.seppur.2020.117593.

[71] N. O'Reilly, N. Giri, S. L. James, Chem. Eur. J. 2007, 13, 3020-3025. DOI: 10.1002/chem.200700090.

[72] N. Giri, C. E. Davidson, G. Melaugh, M. G. Del Pópolo, J. T. A. Jones, T. Hasell, A. I. Cooper, P. N. Horton, M. B. Hursthoused, S. L. James, Chem. Sci. 2012, 3, 2153-2157. DOI: 10.1039/c2sc01007k

[73] J. Zhang, S.-H. Chai, Z.-A. Qiao, S. M. Mahurin, J. Chen, Y. Fang, S. Wan, K. Nelson, P. Zhang, S. Dai, Angew. Chem. 2015, 54, 932-936. D0I: 10.1002/ ange.201409420

[74] G. Melaugh, N. Giri, C. E. Davidson, S. L. James, M. G. Del Pópolo, Phys. Chem. Chem. Phys. 2014, 16, 9422-9431. D0I: 10.1039/C4CP00582A.
[75] S. L. James, Adv. Mater. 2016, 28, 5712-5716. DOI: 10.1002/adma.201505607.

[76] A. Bavykina, A. Cadiau, J. Gascon, Coord. Chem. Rev. 2019, 386, 85-95. DOI: 10.1016/j.ccr.2019.01.015.

[77] N. Giri, M. G. Del Pópolo, G. Melaugh, R. L. Greenaway, K. Rätzke, T. Koschine, L. Pison, M. F. C. Gomes, A. I. Cooper, S. L. James, Nature 2015, 527, 216-220. DOI: 10.1038/nature16072.

[78] R. L. Greenaway, D. Holden, E. G. B. Eden, A. Stephenson, C. W. Yong, M. J. Bennison, T. Hasell, M. E. Briggs, S. L. James, A. I. Cooper, Chem. Sci. 2017, 8 2640-2651. DOI: 10.1039/C6SC05196K.

[79] Z. Deng, W. Ying, K. Gong, Y. Zeng, Y. Yan, X. Peng, Small 2020, 16, 1907016 DOI: 10.1002/smll.201907016.

[80] P. Li, H. Chen, J. A. Schott, B. Li, Y. Zheng, S. M. Mahurin, D.-en Jiang, G. Cui, X. Hu, Y. Wang, L. Li, S. Dai, Nanoscale 2019, 11, 1515-1519. DOI: 10.1039/C8NR07337F.

[81] H. Liu, B. Liu, L.-C. Lin, G. Chen, Y. Wu, J. Wang, X. Gao, Y. Lv, Y. Pan, X. Zhang, X. Zhang, L. Yang, C. Sun, B. Smit, W. Wang, Nat. Commun. 2014, 5 , 5147. DOI: 10.1038 /ncomms6147.

[82] M. C. Gomes, L. Pison, C. Červinka, A. Padua, Angew. Chem. Int. Ed. 2018, 57, 11909-11912. DOI: 10.1002/anie.201805495.

[83] W. Shan, P. F. Fulvio, L. Kong, J. A. Schott, C.-L. D.-Thanh, T. Tian, X. Hu, S. M. Mahurin, H. Xing, S. Dai, ACS Appl. Mater. Interfaces 2018, 10, 32-36. DOI 10.1021/acsami.7b15873.

[84] R. E. Mow, A. S. Lipton, S. Shulda, E. A. Gaulding, T. Gennett, W. A. Braunecker, J. Mater. Chem. A 2020, 8, 23455-23462. D0I: 10.1039/D0TA06768G.

[85] J. Cahir, M. Y. Tsang, B. Lai, D. Hughes, M. A. Alam, J. Jacquemin, D. Rooneya, S. L. James, Chem. Sci. 2020, 11, 2077-2084. DOI: 10.1039/C9SC05770F.

[86] J. Avila, L. F. Lepre, C. C. Santini, M. Tiano, S. D.-Quanquin, K. C. Szeto, A. A. H. Padua, M. C. Gomes, Angew. Chem. Int. Ed. 2021, 60, 12876-12882. DOl: 10.1002/anie.202100090.

[87] B. D. Egleston, K. V. Luzyanin, M. C. Brand, R. Clowes, M. E. Briggs, R. L. Greenaway, A. I. Cooper, Angew. Chem. Int. Ed. 2020, 59, 7362-7366. DOI: 10.1002/anie.201914037.

[88] A. Knebel, A. Bavykina, S. J. Datta, L. Sundermann, L. G.-Tovar, Y. Lebedev, S. Durini, R. Ahmad, S. M. Kozlov, G. Shterk, M. Karunakaran, I. D. Carja, D. Simic, I. Weilert, M. Klüppel, U. Giese, L. Cavallo, M. Rueping, M. Eddaoudi, J. Caro, J. Gascon, Nat. Mater. 2020, 19, 1346-1353. D0I: 10.1038/s41563-020-0764-y.

[89] B. Lai, J. Cahir, M. Y. Tsang, J. Jacquemin, D. Rooney, B. Murrer, S. L. James, ACS Appl. Mater. Interfaces 2021, 13, 932-936. DOI: 10.1021/acsami.0c19044.

[90] J. Avila, C. Červinka, P. Dugas, A. A. H. Pádua, M. C. Gomes, Adv. Mater. Interfaces 2021, 8, 2001982. DOI: 10.1002/admi.202001982.

[91] F. Zhang, F. Yang, J. Huang, B. G. Sumpter, R. Qiao, J. Phys. Chem. B 2016 120, 7195-7200. DOI: 10.1021/acs.jpcb.6b04784.

[92] M. Z. Ahmad, A. Fuoco, Curr. Res. Green Sustain. Chem. 2021, 4, 100070. DOI: 10.1016/j.crgsc.2021.100070.

\section{Leila Moura}

QUILL, Queen's University Belfast, School of Chemistry and Chemical Engineering. Leila Moura obteve o Mestrado em Química pelo Instituto Superior Técnico, em 2008, e o doutoramento pela Université Claude Bernard - Lyon 1, em 2014, seguido de quatro anos de pós-doutoramento, incluindo uma Marie Skłodowska-Curie Individual Fellowship. Começou a sua carreira independente em 2019 com uma Royal Academy of Engineering Research Fellowship na
Queen's University Belfast e é membro do QUILL, Queen's University Belfast Ionic Liquid Laboratories. Os interesses científicos situam-se na área do desenvolvimento e teste de materiais sustentáveis que podem reduzir o impacto ambiental e energético das operações atuais, especificamente na separação de gases e purificação de água. I.moura@qub.ac.uk ORCID.org/0000-0002-7938-5892 